\title{
Adaptación acústica del canto de Turdus leucomelas \\ (Passeriformes: Turdidae) a diferentes niveles de ruido antrópico, en el área metropolitana de Belém, Pará, Brasil
}

\author{
Solange Mendes ${ }^{1}$, Víctor J. Colino-Rabanal ${ }^{2} \&$ Salvador J. Peris* $^{*}$ \\ Dpto. Zoología, Facultad de Biología, Universidad de Salamanca, 37071 Salamanca, España; \\ solangemendes@msn.com,vcolino@usal.es,peris@usal.es \\ * Correspondencia
}

Recibido 15-VII-2016. Corregido 24-I-2017. Aceptado 22-II-2017.

\begin{abstract}
Acoustic adaptation in Turdus leucomelas (Passeriformes: Turdidae) songs to different levels of anthropogenic noise, in the metropolitan area of Belem, Para, Brazil. Anthropogenic noise in urban environments is a major challenge for those species that depend on the transmission of acoustic signals to communicate. To avoid being masked by background noise, some bird species are able to make adjustments in their songs. Studies on vocal adjustment for tropical birds are still scarce and are of interest since both the urban structure and the vegetation associated with urban habitats differ significantly with respect to the cities of temperate climates. In this research we studied the changes in the song parameters of the pale-breasted thrush (Turdus leucomelas) in an urban environment of the metropolitan area of Belém (Brazil). To this end, bird songs were recorded and ambient noise was measured between September and November 2008, in three different acoustic environments (urban, suburban and rural) along an urban gradient. The songs of 12 individuals per area were selected (a total of 36). Possible differences between song parameters were analyzed by ANOVAs. To assess the noise impact on bird song, we only considered the spectrum of environmental noise within the range of vocalizations of the species. In general, birds of urban habitats presented songs with higher maximum frequencies and with a wider range of notes, than their counterparts in suburban and rural areas. The differences were more pronounced in relation to rural areas. No differences in the minimum frequencies, the concentration of energy, or the average duration of the notes were found. These results differ from other studies and could possibly indicate variations in the way birds try to succeed in habitats with high ambient noise. It is necessary further exploration on the role of these changes in the effective improvement of intra-specific communication for the species in such environments. Rev. Biol. Trop. 65 (2): 633-642. Epub 2017 June 01.
\end{abstract}

Key words: anthropogenic noise, bioacoustics, bird song, urban gradient, vocal adjustment.

Los estudios sobre el efecto del ruido antrópico en las poblaciones de aves silvestres se orientan a identificar y cuantificar los impactos sobre la dinámica poblacional, conducta, comunicación u otros procesos de socialización (Slabbekoorn \& Boer-Visser, 2006; Wood \& Yezerinac, 2006). Los medios urbanos generan entornos acústicos caracterizados por niveles elevados de ruido ambiental que dificultan la comunicación vocal y que constituyen un reto para aquellas especies que dependen de la vocalización en sus relaciones intra-específicas.
En general, el aumento del ruido ambiental por tráfico tiende a provocar una disminución tanto en la riqueza de especies de aves, como en sus tamaños poblacionales (Zande, Keurs, \& Weijden, 1980; Reijnen, Foppen, \& Veenbaas, 1997; Weiserbs \& Jacob, 2001; Peris \& Pescador, 2004; Slabbekoorn \& Ripmeester, 2008). Sin embargo, algunas especies muestran un considerable grado de adaptación a estos entornos, sugiriendo que la plasticidad del canto facilita ajustes vocales que contribuyen a reducir el enmascaramiento por ruido 
ambiental (Fernández-Juricic, 2001; Rheindt, 2003; Slabbekoorn \& Peet, 2003; Brumm, 2004; Fernández-Juricic et al., 2005; Parris \& Schneider, 2009). Esta plasticidad favorece la comunicación intraespecífica (Brumm \& Slabbekoorn, 2005) a pesar del elevado coste energético (Suthers, Goller, \& Pytte, 1999), y podría acelerar la divergencia evolutiva hacia una especialización urbana (Slabbekoorn, 2013) a pesar del ruido de fondo (Harms, Fleming, \& Stoskopf, 1997).

La adaptación al entorno urbano se explicaría por varias hipótesis. Aquí se investiga la de adaptación acústica. Dicha hipótesis sostiene que, por selección ambiental, los cantos presentan una estructura que favorece una maximización de la transmisión bajo las condiciones ambientales en que se generan (Morton, 1975). La adaptación del canto a las características acústicas ambientales aparece y se mantendría mediante aprendizaje; copiando los individuos jóvenes aquellos cantos - no filtrados por el ambiente- que mejor perciben de sus con-específicos (Mendes, Colino-Rabanal, \& Peris, 2011a).

Los estudios sobre ajustes vocálicos para aves tropicales son aún escasos y resultan de interés, ya que tanto la estructura urbana como la propia vegetación asociadas a los hábitats urbanos, difieren de forma notable respecto a las ciudades de climas templados. En esta investigación se analizan algunos parámetros físicos del canto en una especie de amplia distribución neotropical, el Zorzal Sabiá (Turdus leucomelas, Vieillot 1818, Turdidae) bajo la hipótesis de adaptación acústica. El éxito en un entorno urbano estaría ligado a la capacidad de adaptación a este nuevo hábitat. Para analizar dicha adaptación, exploramos, para una área metropolitana tropical, los cambios en el canto de la especie que le permiten comunicarse en un entorno acústico característico del medio urbano (McKinney, 2006). Dichas variaciones mostrarían cierta adaptación a las condiciones ambientales antrópicas (Ríos-Chelén, Salaberria, Barbosa, Macías-García, \& Gil, 2012).

\section{MATERIALES Y MÉTODOS}

T. leucomelas está bien extendido en el Neotrópico, nidificando desde el norte de Colombia hasta norte de Argentina (Macedo et al., 2007). Nidifica en cualquier hábitat, incluyendo zonas urbanas. Exhibe una amplia gama de vocalizaciones; desde simples notas de llamada hasta cantos elaborados, pero siempre con patrones sencillos. Como otros zorzales, en temporada reproductora emite cantos largos de 20-30 min al amanecer (4:00-6:00 h.l.), seguidos por un intervalo inactivo. Durante el resto del día emiten cantos cortos de menor duración, hasta otro máximo vocal al anochecer. El canto completo consiste en distintas secuencias de sonidos, con notas en que predominan sonidos puros modulados, intercalando notas sin modulación o sonidos con trinos. Cada frase tiene una duración media de 3-4 s, separadas por pausas en torno a $3 \mathrm{~s}$, y dando una cadencia cercana a $6 \mathrm{~s}$. Las frases pueden ser complejas o simples. La especie suele cantar partes "motivo" repitiendo las mismas notas en cada una de ellas. Raras veces realiza gorjeos o "twitter", siempre con notas más cortas que las de las "motivo". En cortejo o persecución, cambian el orden de las frases, tornándose confusas e incompletas, similar a otros zorzales (Dalbelsteen \& Pedersen, 1992). El rango de frecuencia se sitúa entre 1000 y $4000 \mathrm{~Hz}$, como la mayoría de Passeriformes.

Área de estudio: Las grabaciones se efectuaron en el área metropolitana de Belém de Pará (Brasil), ciudad a orillas del río Guamá $\left(1^{\circ} 27^{\prime} 28^{\prime \prime} \mathrm{S}-48^{\circ} 30^{\prime} 06^{\prime \prime} \mathrm{W}\right)$. Las grabaciones se localizaron en un radio de $100 \mathrm{~km}$ en torno a la ciudad. Se diferenciaron 3 zonas: a) "área urbana (U)": si se encuentra en un radio inferior a $20 \mathrm{~km}$ del centro de la ciudad y se caracteriza por zonas ajardinadas, oficinas y elevada intensidad de tráfico; b) "área periurbana $(\mathrm{P})$ ": entre 20 y $30 \mathrm{~km}$ del centro, con jardines, remanentes de vegetación natural, viviendas unifamiliares y tráfico local; c) "área rural (R)": entre 30-100 km de distancia al centro, es el área con menor contaminación acústica, 
con escasa actividad antrópica, presencia de bosquetes urbanos, mosaicos de frutales y palmeras, y fragmentos de bosque original. Las tres zonas cuentan con zonas verdes con vegetación amazónica, y plantas exóticas como Mangifera indica, que forma parte de la dieta del ave.

Grabación de cantos: Las grabaciones se tomaron entre septiembre y noviembre de 2008, coincidiendo con la reproducción del T. leucomelas. Los cantos se grabaron con un micrófono unidireccional Sennheiser ME66 (con adaptador K6 Sennheiser) y una grabadora digital Marantz PMD 671, configurada para PCM-48kHz-24 bits, y una velocidad en bits de $768 \mathrm{kbps}$ para obtener una señal de audio de alta calidad. La grabadora se configuró con una especificación técnica del rango dinámico equivalente al del medidor de nivel sonoro utilizado (94 dB), con baja distorsión harmónica total (THD) (0.03\% de pérdida por entrada del micrófono XLR). El equipo contaba con un protector de viento MZW 415, y un auricular "Sennheiser" para comprobar la entrada de señales de audio.

Las grabaciones se realizaron durante el periodo de mayor actividad para la especie, entre 4:00-7:00 horas. En total, se emplearon 400 horas en campo, con un total de 75 grabaciones de diferentes individuos. Para evitar el exceso de ruido de fondo que pudiera interferir en el posterior análisis, sólo se realizaron grabaciones en días sin lluvia y velocidad del viento inferior a $3 \mathrm{~m} / \mathrm{s}$ (medido por anemómetro). Las grabaciones se realizaron a $8-50 \mathrm{~m}$ de distancia de cada individuo, ajustando el receptor de la grabadora según distancia.

Para el análisis, se escogieron al azar sólo aquellas grabaciones de mayor duración ( $>5$ minutos), sin interferencias con otros ruidos o superposición de cantos de otras especies aviares. Se seleccionaron 12 vocalizaciones de distintos individuos por cada zona del gradiente (U, P, R), totalizando 36 grabaciones distintas. Las condiciones de grabación fueron similares en cada ambiente, sin diferencias aparentes entre hábitats y calidad de grabación.
Como los parámetros de canto se ven influidos por la densidad poblacional (Hamao, Watanabe, \& Mori, 2011), se realizó en cada grabación una estimación poblacional. Desde el punto de grabación, se estimó el número de individuos vistos u oídos, teniendo precaución de evitar dobles conteos. Posteriormente, se calculó el tamaño del área prospectada, estimando el número de individuos por hectárea (ha). El sistema es comparable al método de punto fijo de conteo (Ralph et al., 1996).

Medición de los niveles de presión sonora: Para analizar la relación entre los niveles de ruido ambiental y los parámetros del canto en cada punto de muestreo $(n=36)$, se cuantificó el ruido de fondo. Las mediciones se realizaron con un sonómetro Clase I, modelo Brüel y Kjaer 2238E, un micrófono con capacidad 1/2" modelo ByK 4188 y filtros de $1 / 1$ y 1/3 de octavas (Mendes, Cavalcante, Colino-Rabanal, \& Peris, 2010). El tiempo de medición fue de 10 minutos en cada punto de muestreo. El medidor se montaba sobre un trípode a una altura de 1.5 $\mathrm{m}$ del suelo y a $2 \mathrm{~m}$ de distancia de cualquier obstáculo que pudiera proyectar sombra acústica o reflejar energía sonora.

Como nivel de ruido no se consideró el nivel de presión sonora en su conjunto, sino sólo aquel situado dentro del rango de frecuencia de vocalización del $T$. leucomelas, el único que causaría interferencias en su comunicación (Cavalcante, Mendes, Corbo, \& Schwabe, 2008). Para el análisis del espectro de frecuencias, nos remitimos a Mendes et al. (2011a). A partir de las mediciones, se elaboró un descriptor acústico $L_{e q(T l e)}$ que expresa los niveles sonoros en el rango de vocalización de la especie para cada punto muestreado:

$$
\begin{gathered}
\mathrm{L}_{T l e}=10 \cdot \log \left(10^{\frac{\mathrm{L}_{\mathrm{eq}(\mathrm{N} 1)}}{10}}+10^{\frac{\mathrm{L}_{\mathrm{eq}(\mathrm{N} 2)}}{10}}+\ldots+10^{\frac{\mathrm{L}_{\mathrm{eq}(\mathrm{N} n)}}{10}}\right) \\
\mathrm{L}_{e q(T l e)}=10 \cdot \log \left(\frac{1}{\mathrm{n}} \cdot\left(10^{\frac{\mathrm{L}_{\mathrm{eq}(\mathrm{N} 1)}}{10}}+10^{\frac{\mathrm{L}_{\text {eq }(\mathrm{N} 2)}}{10}}+\ldots+10^{\frac{\mathrm{L}_{\mathrm{eq}(\mathrm{Nn})}}{10}}\right)\right)
\end{gathered}
$$

en donde $L_{e q(T l e)}$ es el nivel de presión sonora equivalente y lineal dentro del rango de vocalización del T. leucomelas en bandas de tercio de octavas entre $1.6 \mathrm{kHz}$ y $8 \mathrm{kHz}$. $N$ es el valor 
de presión sonora dentro del rango de vocalizaciones del ave en cada medición individual, y $n$ el número de mediciones realizadas a intervalos de tiempo iguales para obtener el nivel de presión sonora.

Análisis acústico y estadístico: Para el análisis de los parámetros del canto, se empleó el software Avisoft SASLab (Spetch, 1998) y los cálculos del nivel de presión sonora de acuerdo a Mendes et al. (2011a). El ruido de fondo de las grabaciones se eliminó mediante filtros FFT (Baker \& Logue, 2003). Para los parámetros del canto, se cuantificó la duración, el número de notas, las frecuencias mínimas y máximas, la distribución de energía vocal dentro del rango de frecuencias de cada individuo (Zollinger, Podos, Nemeth, Goller, \& Brumm, 2012), y la entropía o diversidad vocal (Silva, Piqueira, \& Vielliard, 2000).

El cálculo de entropía está basado en la teoría de la información (Raisbeck, 1963; Beecher, 1989). Se designa un alfabeto para el canto de la especie. Cada individuo posee un repertorio compuesto por $\mathrm{N}$ símbolos (notas). Se calcula la frecuencia de aparición de cada tipo de nota de su repertorio, esto es, se obtiene una probabilidad $\mathrm{p}_{\mathrm{i}}$ asignada a cada símbolo $\left(\mathrm{I}_{\mathrm{i}}\right)$ en función de su frecuencia relativa. Con ello, se calcula la entropía/diversidad (E) individual.

Las pruebas de Levene y de KolmogorovSmirnov confirmaron la normalidad de los datos, permitiendo analizar las posibles diferencias en los parámetros vocales entre las tres zonas mediante un análisis de la varianza (ANOVA) y los posteriores análisis post-hoc mediante el test de Scheffé. Los niveles de presión sonora entre hábitats se compararon con un ANOVA (Fowler \& Cohen, 1999). El coeficiente de correlación de Pearson se empleó para determinar la relación entre los niveles de ruido de fondo y los parámetros de los cantos. El nivel de significación fue $\mathrm{P}<$ 0.05 . Los análisis estadísticos fueron realizados con SPSS 17.0.

\section{RESULTADOS}

Niveles de presión sonora y densidades poblacionales en los tres hábitats: Las diferencias en presión sonora en el rango de vocalización del T. leucomelas $L_{e q(T l e)}$ entre los tres tipos de hábitats (Urbano, Periurbano y Rural) fueron altamente significativas $\left(\mathrm{F}_{2,35}=61.69 ; \mathrm{P}\right.$ $<0.01)$. En el análisis post-hoc se constató que hay diferencias entre todas las zonas. Como era de esperar, las zonas urbanas (con 59.44 $\mathrm{dB}$ en promedio) eran más ruidosas y variables en sonidos que las periurbanas $(45.02 \mathrm{~dB})$. El ruido fue menor en las áreas rurales $(36.94 \mathrm{~dB}$ ) (Fig. 1). No hubo diferencias estadísticamente significativas entre las poblaciones del T. leucomelas en los tres hábitats $\left(\mathrm{F}_{2.35}=0.97\right.$ y $\mathrm{P}=$ 0.40). La media de individuos observados en zona urbana fue similar a la observada en la rural, siendo sólo algo menores las poblaciones de la zona periurbana.

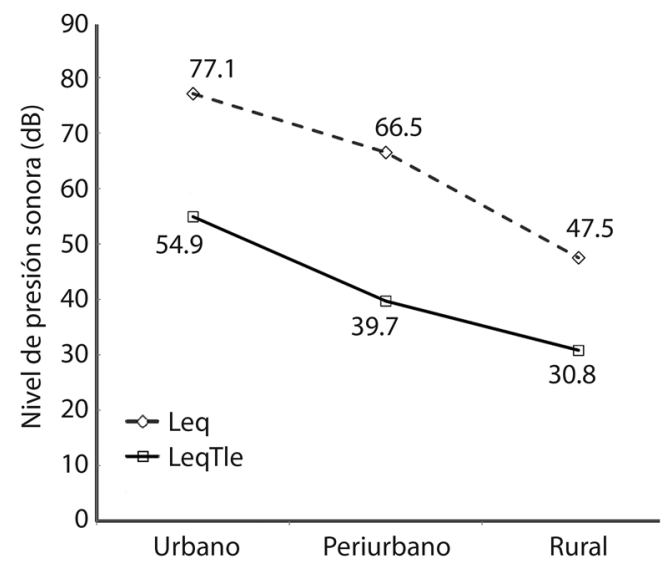

Fig. 1. Niveles de presión sonora en las tres zonas de estudio $(\mathrm{U}, \mathrm{P}, \mathrm{R})$ expresada en escala lineal. $\mathrm{L}_{\mathrm{eq}}$ : Presión sonora total del ambiente; $\mathrm{L}_{\mathrm{eq}(\mathrm{Tle})}$ : Nivel de presión sonora limitado al rango de comunicación, entre $1.5-4.0 \mathrm{kHz}$, del T. leucomelas.

Fig. 1. Sound pressure levels in the three areas of study (U, $\mathrm{P}, \mathrm{R})$ expressed in linear scale. $\mathrm{L}_{\mathrm{eq}}$ : Total sound pressure in the environment; $\mathrm{L}_{\mathrm{eq}(\mathrm{Tle})}$ : Sound pressure levels, from 1.5 to $4.0 \mathrm{kHz}$, according to the vocal range of T. leucomelas. 
Diferencias en la diversidad de canto entre hábitats: Los individuos de zonas rurales mostraron una diversidad menor de canto que los procedentes de zonas urbanas y periurbanas $\left(\mathrm{F}_{2.35}=4.56 ; \mathrm{gl}=1, \mathrm{P}=0.04\right)$ (Fig. 2). Por otro lado, no se detectaron diferencias significativas entre la zona urbana y la periurbana $\left(\mathrm{F}_{2.35}=2.49 ; \mathrm{gl}=2, \mathrm{P}=0.13\right)$. Se encontró una correlación significativa entre los niveles de presión sonora dentro de cada hábitat y la diversidad del canto $(r=0.326, p=0.05)$.

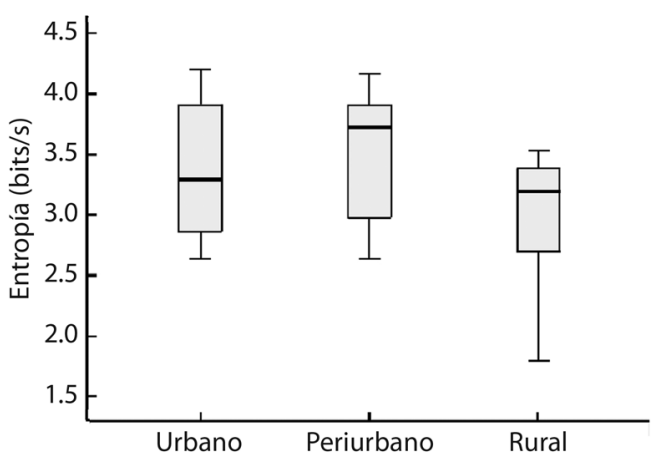

Fig. 2. Entropía del canto del T. leucomelas según hábitat. Fig. 2. Song entropy in T. leucomelas according to habitat.

Diferencias en el rango de frecuencias: Las frecuencias máximas variaron según hábitat, siendo más altas en ambientes urbanos y bajando gradualmente en periurbanos y rurales $\left(\mathrm{F}_{2.35}=3.93 ; \mathrm{P}=0.02\right)$. El test post-hoc constató diferencias significativas entre ambientes urbanos y rurales (Fig. 3A, Fig. 3B). Sin embargo, en frecuencias mínimas no habría variación entre zonas $\left(\mathrm{F}_{2.35}=0.48 ; \mathrm{P}=0.62\right)$. Hubo una correlación estadísticamente significativa $(r=0.436, P<0.001)$ entre niveles de presión sonora $(\mathrm{dB})$ del hábitat y frecuencias máximas del canto.

Variaciones de la concentración de energía del canto: El intervalo de frecuencias de vocalización del T. leucomelas se situó entre $1500 \mathrm{~Hz}$ y 4000 Hz. Dentro del hábitat urbano, se observó que $50 \%$ de la concentración de energía de los cantos se concentraba en torno
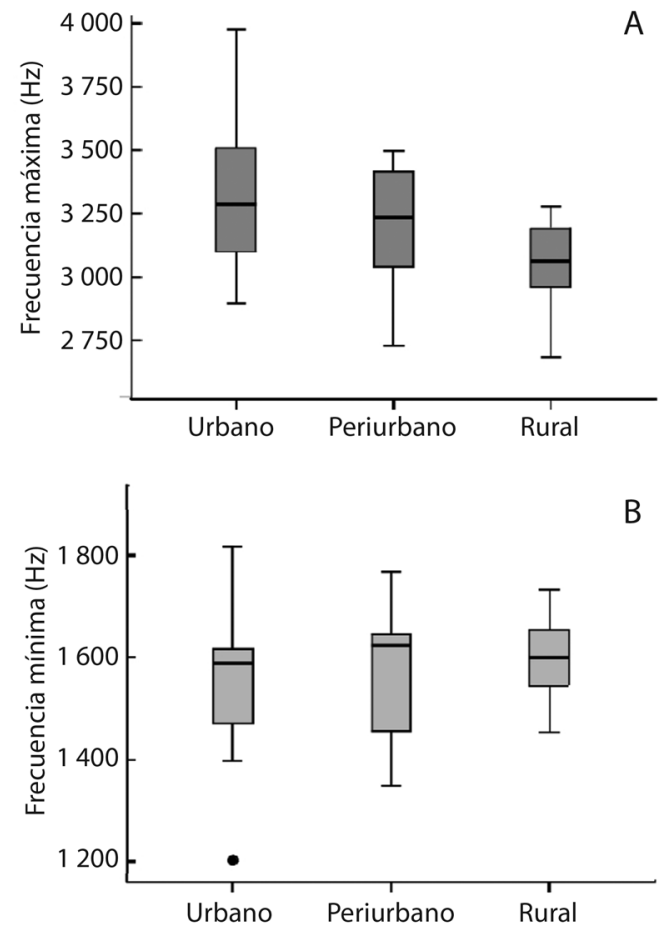

Fig. 3. Frecuencias máximas (A) y mínimas (B) del canto de 36 individuos de T. leucomelas agrupados por hábitats. Fig. 3. Maximum (A) and minimum (B) frequencies from 36 T. leucomelas's birds according to habitat.

a $2300 \mathrm{~Hz}$. En entornos periurbanos y rurales estuvieron por debajo de este valor (Fig. 4), aunque las diferencias no fueron significativas $(\mathrm{r}=-0.012, \mathrm{P}=0.84)$. Tampoco hubo correlación entre la concentración de energía y el ruido ambiental $(\mathrm{r}=-0.068, \mathrm{P}=0.69)$.

Variaciones de la duración de notas: No se encontraron diferencias significativas entre la duración de las notas y el tipo de hábitat $\left(\mathrm{F}_{2.35}=0.11, \mathrm{P}=0.89\right)$ (Fig. 5) ni hubo correlación entre ambas $(\mathrm{r}=-0.129, \mathrm{P}=0.45)$.

\section{DISCUSIÓN}

Los resultados sugieren diferencias vocales en la diversidad y frecuencia del canto entre T. leucomelas procedentes de zonas urbanas, periurbanas y rurales. Especialmente, hay una mejora en las llamadas de reconocimiento 


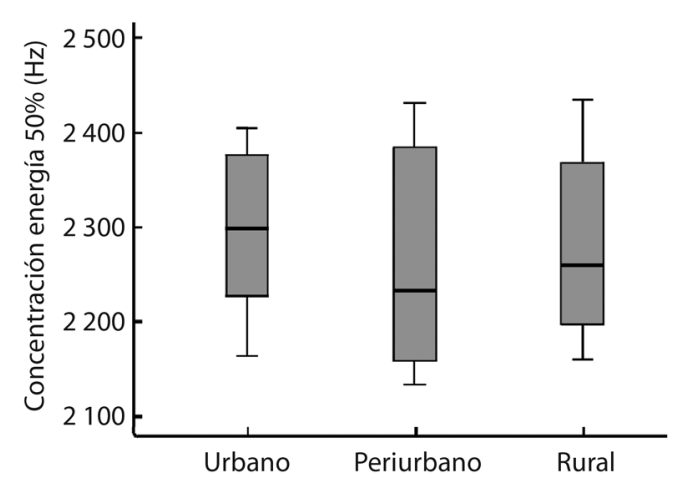

Fig. 4. Energía vocal emitida por el T. leucomelas según hábitat.

Fig. 4. Vocal energy emitted by T. leucomelas according to habitat.

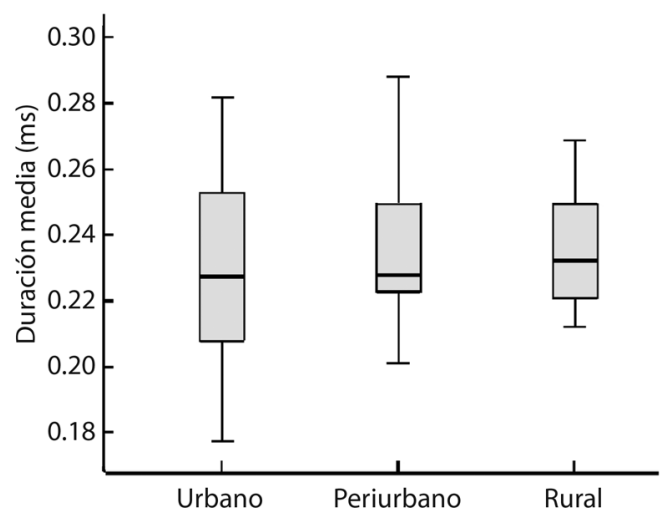

Fig. 5. Duración media de notas del T. leucomelas en milisegundos (ms) según hábitat.

Fig. 5. Mean duration (ms) from T. leucomelas's notes according to habitat.

por repetición frecuente del canto, ajustando la amplitud de la vocalización para sobreponerse al ruido de fondo en áreas ruidosas. Estas adaptaciones estarían en función del ruido antrópico, un factor generador de posibles cambios en los cantos de aves canoras (Dooling, 1982; Hu \& Cardoso, 2010; Redondo, Barrantes, \& Sandoval, 2013).

Así, los T. leucomelas urbanos desarrollan cantos más versátiles, con frecuencias máximas más elevadas y una mayor diversidad que los rurales. Por el contrario, Rios-Chelen et al. (2012) no observaron diferencias en frecuencias máximas en la misma especie, aunque si en las frecuencias mínimas. Otras especies aviares muestran menor diversidad vocal en ambientes urbanos (Potvin, Mulder, \& Parris, 2014), o bien aumentan las frecuencias mínimas (Hanna, Blouin-Demers, Wilson, \& Mennill, 2011), demostrando diferentes estrategias en aves urbanas.

En general, cuanto más versátil sea el canto, mayor será la capacidad de comunicación que tendrá una especie (Dingle, 1972). Las señales comunicativas evolucionan para aumentar la eficiencia en la transmisión de la información, ya sea un incremento información, un menor coste energético o un menor tiempo necesario (Dawkins \& Krebs, 1978). Los $T$. leucomelas periurbanos vocalizan con un promedio de diversidad mayor que los individuos urbanos y rurales. Algunas de las grabaciones de los cantos en zonas periurbanas, las tomadas entre las 6:00 y 7:00 horas, coincidirían cuando los niveles de ruido antropogénico empiezan a incrementarse -inicio de horario laboral- lo que explicaría la mayor versatilidad de cantos. Mientras, los individuos urbanos suelen iniciar las vocalizaciones en horas previas, entre las 4:00 y 5:00 horas, cuando los niveles de ruido ambiental son más bajos. Esta conducta estaría regulada por un ciclo circadiano exógeno, basado en longitud de los días (Gwinner \& Brandstätter, 2001) y en la iluminación artificial en zonas urbanas, que provocaría alteraciones del canto, favoreciendo los tempranos o nocturnos (Cassone \& Menaker, 1984; Brandstätter, 2003; Beecher \& Brenowitz, 2005).

Otras especies como el ruiseñor europeo Luscinia megarhynchos aumentan la amplitud vocal e intensidad del canto en ambientes ruidosos, requiriendo para ello un mayor gasto energético (Brumm, 2006). Comparando los costes metabólicos en la vocalización de Paseriformes, Oberweger y Goller (2001) demostraron que un incremento de $16 \mathrm{~dB}$ del canto del estornino pinto Sturnus vulgaris, conlleva un aumento del $16 \%$ en consumo de oxígeno. La concentración de energía del canto del T. leucomelas no presenta excesivas variaciones entre distintos tipos de hábitats, 
concentrándose en 2200-2300 Hz. Sin embargo, un cambio de frecuencias, aún leve, supone mayor gasto de energía, y el aumento de frecuencias en respuesta al ruido implicaría un coste, al requerir mayor contracción muscular de la siringe (Suthers et al. 1999), o limitar los rasgos de cantos seleccionadas en atracción sexual (Slabbekoorn \& Ripmeester, 2008). Así, el gorrión melódico Melospiza melodia aumenta las frecuencias más bajas y su canto presenta menor energía en áreas ruidosas dentro de los $1400 \mathrm{~Hz}$, sin diferencias en las frecuencias altas, esto es, su ajuste vocal se produce dentro del ancho de banda enmascarada por el ruido ambiental (Wood \& Yezerinac, 2006).

Respecto a cambios vocales según densidad poblacional, el T. leucomelas es abundante en todas las áreas estudiadas, y sus diferencias poblacionales no incidirían en cambios vocales entre los individuos. La ciudad presenta niveles de ruido elevados, pero la diversidad de árboles y superficie de los parques podrían servir de atenuación mediante recursos tróficos y de nidificación, haciendo la ciudad favorable para la especie. Al ser una especie omnívora, la riqueza de frutos en parques y otros alimentos de origen antrópico, explicarían una población estable con buen éxito de cría (Luniak, Mulsow, \& Walasz, 1990), un aumento del sedentarismo (Partecke \& Gwinner, 2007), y una prolongada temporada reproductora (Partecke, Van't Hof, \& Gwinner, 2004); aspectos que ocurren en otras especies de Turdidae (Mendes, Colino-Rabanal, \& Peris, 2011b), e influirían en la adaptación del T. leucomelas en centros urbanos.

Para evitar el enmascaramiento de cantos por altos niveles de ruido, diversas especies introducen cambios en sus señales acústicas: frecuencias, amplitudes, estructuras temporales y momentos (Patricelli \& Blickley, 2006). En el $T$. leucomelas, se detectan ajustes en frecuencias máximas, pero no en mínimas. Quizá, y al menos en esta especie, este ajuste permita obtener mayor alcance vocal por encima de la frecuencia del ruido ambiental, según la hipótesis de adaptación acústica (Chappuis, 1971; Slabbekoorn, 2004). Esto podría revelar cierta plasticidad vocal de la especie en áreas urbanas, sin los efectos perjudiciales que sufren otras especies aviares (Arroyo-Solís, Castillo, Figueroa, López-Sánchez, \& Slabbekoorn, 2013; Halfwerk, Holleman, Lessells, \& Slabbekoorn, 2011; Colino-Rabanal, Mendes, Peris, \& Pescador, 2016).

Como conclusión, las perturbaciones sonoras de origen antrópico inducen cambios la vocalización de T. leucomelas. En ambientes urbanos emiten cantos más diversos y con mayor frecuencia que en áreas periurbanas y rurales. Aunque los datos no son significativos, hay una tendencia a que las aves urbanas inviertan más energía en las vocalizaciones. No hay diferencias significativas en duración de las notas entre zonas. Las poblaciones de aves entre las tres áreas es similar, y el ruido no afectaría al número de individuos por hábitat.

\section{AGRADECIMIENTOS}

Milena Corbo y Leiliane Moura ayudaron en campo; M. Silva y J. Vielliard (+) por sugerencias en acústica. El Dpto. de Vibración y Acústica de la Universidad Federal do Pará (G. S. V. de Melo y L. Bastos) por el equipo B\&K. Fátima acompañó en campo y la familia Aldanas - Dolores y Trini- por su hospitalidad. A dos revisores anónimos y Ríos-Chelén por sus sugerencias. Por ayuda parcial, a la Fundación "Samuel Solórzano Barruso" (FS/7-2011).

\section{RESUMEN}

El ruido antropogénico en entornos urbanos supone un importante reto para aquellas especies que dependen de la transmisión de señales acústicas para comunicarse. Para evitar ser enmascaradas por el ruido de fondo, algunas especies de aves son capaces de realizar ajustes vocálicos en sus cantos. Los estudios sobre ajustes vocálicos para aves tropicales son aún escasos y resultan de interés ya que tanto la estructura urbana como la propia vegetación asociadas a los hábitats urbanos difieren de forma notable respecto a las ciudades de climas templados. En este estudio se pretende describir posibles cambios en los parámetros de canto del zorzal sabiá (Turdus leucomelas) en un entorno urbano, el área metropolitana de Belém (Brasil). Para ello, se grabaron los cantos de la especie entre septiembre y noviembre 2008 y se midió el ruido ambiental 
en tres diferentes ambientes acústicos (urbano, periurbano y rural) a lo largo de un gradiente urbano. Se seleccionaron los cantos de 12 individuos por cada ambiente (un total de 36). Las posibles diferencias entre los parámetros del canto se analizaron mediante ANOVAs. Para evaluar el impacto acústico sobre el canto, sólo se tuvo en cuenta el espectro del ruido ambiental dentro del rango de vocalización de la especie. En general, las aves de hábitats urbanos presentaron cantos con frecuencias máximas más elevadas y con una mayor diversidad de notas que sus congéneres de zonas periurbanas y rurales. Las diferencias fueron más acusadas en relación a las zonas rurales. No se encontraron diferencias en cuanto a las frecuencias mínimas, la concentración de la energía, o la duración media de las notas. Estos resultados difieren de otros estudios y quizás podrían indicar variaciones en la forma en la que las aves intentan desenvolverse en hábitats con un elevado ruido ambiental. Debe profundizarse sobre el papel de estas modificaciones en la mejora efectiva de la comunicación intra-específica para la especie en dichos ambientes.

Palabras clave: ruido antrópico, bioacústica, canto aves, gradiente urbano, ajuste vocálico.

\section{REFERENCIAS}

Arroyo-Solís, A., Castillo, J. M., Figueroa, E., LópezSánchez, J. L., \& Slabbekoorn, H. (2013). Experimental evidence for an impact of anthropogenic noise on dawn chorus timing in urban birds. Journal of Avian Biology, 44, 288-296. DOI: 10.1111/j.1600-048X.2012.05796.X

Baker, M. C., \& Logue, D. M. (2003). Population differentiation in a complex bird sound: a comparison of three bioacoustical analysis procedures. Ethology, 109(3), 223-242. DOI: 10.1046/j.1439-0310.2003.00866.x

Beecher, M. D. (1989). Signaling systems for individual recognition: an information theory approach. Animal Behaviour, 38, 248-261. DOI: 10.1016/ S0003-3472(89)80087-9

Beecher, M. D., \& Brenowitz, E. A. (2005). Functional aspects of song learning in songbirds. Trends in Ecology and Evolution, 20, 143-149. DOI: 10.1016/j. tree.2005.01.004

Brandstätter, R. (2003). Encoding time of day and time of year by the avian circadian system. Journal of Neuroendocrinology, 15(4), 398-404. DOI: 10.1046/j.1365-2826.2003.01003.x

Brumm, H. (2004). The impact of environmental noise on song amplitude in a territorial bird. Journal of Animal Ecology, 73, 434-440. DOI: 10.1111/j.0021-8790.2004.00814.x
Brumm, H. (2006). Animal communication: city birds have changed their tune. Current Biology, 16, 1003-1004. DOI: 10.1016/j.cub.2006.10.043

Brumm, H., \& Slabbekoorn, H. (2005). Acoustic communication in noise. Advances in the Study of Behavior, 35, 151-209. DOI: 10.1016/S0065-3454(05)35004-2

Cassone, V. M., \& Menaker, M. (1984). Is the avian circadian system a neuroendocrine loop? Journal of Experimental Zoology, 232(3), 539-549. DOI: 10.1002/ jez.1402320321

Cavalcante, K. V., Mendes, S., Corbo, M., \& Schwabe, W. K. (2008). Avaliação acústica ambiental de um hábitat urbano do pássaro "Troglodytes aedon" exposto ao ruído de tráfego em Campinas-SP. XXII Encontro Sobrac.

Chappuis, C. (1971). Un example de l'influence du milieu sur les emissions vocales des oiseaux: L'evolution des chants en foret equatoriale. Terre Vie, 118, 183-202.

Colino-Rabanal, V. J., Mendes, S., Peris, S. J., \& Pescador, M. (2016). Does the song of the wren Troglodytes troglodytes change with different environmental sounds? Acta Ornithologica, 51, 13-22. DOI: 10.3161/00016454AO2016.51.1.002

Dalbelsteen, T., \& Pedersen, S. B. (1992). Song features essential for species discrimination and behaviour assessment by male blackbirds (Turdus merula). Behaviour, 121(3-4), 259-287.

Dawkins, R., \& Krebs, J. R. (1978). Animal signals: information or manipulation. En J. R. Krebs \& N. B. Davies (Eds.), Behavioral Ecology (pp. 282-309). Oxford: Blackwell Scientific Publ.

Dingle, H. (1972). Aggressive behaviour in stomatopods and the use of information theory in the analysis of animal communication. En H. E. Winn \& B. L. Olla (Eds.), Behavior of Marine Animals: Current Perspectives in Research. Invertebrates (Vol. 1, pp. 126-156). New York: Plenum.

Dooling, R. J. (1982). Auditory perception in birds. En D. E. Kroodsma \& E. H. Miller (Eds.), Accoustic communication in birds (Vol 1, pp. 95-130). New York: Academic Press.

Fernández-Juricic, E. (2001). Avian spatial segregation at edges and interiors of urban parks in Madrid, Spain. Biodiversity and Conservation, 10, 1303-1316. DOI: 10.1023/A:1016614625675

Fernández-Juricic, E., Poston, R., De Collibus, K., Morgan, T., Bastain, B., Martin, C., Jones, K., \& Treminio, R. (2005). Microhabitat selection and singing behavior patterns of male housefinches (Carpodacus mexicanus) in urban parks in a heavily urbanized landscape in the Western U.S. Urban Habitats, 3, 49-69. 
Fowler, J. \& Cohen, L. (1999). Estadistica básica en Ornitología. Madrid: SEO/BirdLife.

Gwinner, E., \& Brandstatter, R. (2001). Complex bird clocks. Philosophical Transactions of the Royal Society B: Biological Sciences, 356(1415), 18011810. DOI: $10.1098 /$ rstb.2001.0959

Halfwerk, W., Holleman, L. J. M., Lessells, C. M., \& Slabbekoorn, H. (2011). Negative impact of traffic noise on avian reproductive success. Journal of Applied Ecology, 48, 210-219. DOI: 10.1111/j.1365-2664.2010.01914.x

Hamao, S., Watanabe, M., \& Mori, Y. (2011). Urban noise and male density affect songs in the great tit Parus major. Ethology, Ecology \& Evolution, 23, 111-119. DOI: $10.1080 / 03949370.2011 .554881$

Hanna, D., Blouin-Demers, G., Wilson, D. R., \& Mennill, D. J. (2011). Antropogenic noise affects song structure in redwinged blackbirds (Agelaius phoeniceus). Journal of Experimental Biology, 214, 3549-3556. DOI: $10.1242 /$ jeb.060194

Harms, C. A., Fleming, W. J., \& Stoskopf, M. K. (1997). A technique for dorsal subcutaneous implantation of heart rate biometry transmitters in black ducks: application in an aircraft noise response study. Condor, 99, 231-237.

Hu, Y., \& Cardoso, G. C. (2010). Which birds adjust the frequency of vocalizations in urban noise? Animal Behaviour, 79, 863-867. DOI: 10.1016/j. anbehav.2009.12.036

Luniak, M., Mulsow, R., \& Walasz, K. (1990). Urbanization of the European blackbird - Expansion and adaptations of urban populations. En M. Luniak (Ed.), Urban ecological studies in Central Andeastern Europe (pp. 187-198). Warsaw: Polish Academy of Sciences.

Macedo, D. J., Paula, H., Correa, A., Santos, J., Neves, K., \& Pesquero, M. (2007). Guildas alimentares de aves em ambientes de floresta estacional semidecídua e cerradão. En Seminário de iniciação científica (pp. 1-6). Anápolis.

McKinney, M. L. (2006). Urbanization as a major cause of biotic homogenization. Biological Conservation, 127, 247-260. DOI: 10.1016/j.biocon.2005.09.005

Mendes, S., Cavalcante, K. V., Colino-Rabanal, V. J., \& Peris, S. J. (2010). Evaluación del impacto de la Contaminación Acústica en el rango de vocalización de Paseriformes basado en el SIL- "Speech Interference Level”. Revista de Acústica, 41(3-4), 33-41.

Mendes, S., Colino-Rabanal, V. J., \& Peris, S. J. (2011a). Diferencias en el canto de la ratona común (Troglodytes musculus) en ambientes con distintos niveles de influencia humana. Hornero, 26(2), 85-93.
Mendes, S., Colino-Rabanal, V. J., \& Peris, S. J. (2011b). Bird song variations along an urban gradient: the case of the European blackbird (Turdus merula). Landscape and Urban Planning, 99, 51-57. DOI: 10.1016/j. landurbplan.2010.08.013

Morton, E. S. (1975). Ecological sources of selection on avian sounds. American Naturalist, 109, 17-34.

Oberweger, K., \& Goller, F. (2001). The metabolic cost of birdsong production. Journal of Experimental Biology, 204, 3379-3388.

Parris, K. M., \& Schneider, A. (2009). Impacts of traffic noise and traffic volume on birds in roadside habitats. Ecology and Society, 14, 29. http://www.ecologyandsociety.org/vol14/iss1/art29/

Partecke, J., \& Gwinner, E. (2007). Increased sedentariness in European blackbirds following urbanization: a consequence of local adaptation? Ecology, 88, 882890. DOI: $10.1890 / 06-1105$

Partecke, J., Van’t Hof, T., \& Gwinner, E. (2004). Differences in the timing of reproduction between urban and forest European blackbirds (Turdus merula): result of phenotypic flexibility or genetic differences? Proceedings of the Royal Society B: Biological Sciences, 271, 1995-2001. DOI: 10.1098/rspb.2004.2821

Patricelli, G. L., \& Blickley, J. L. (2006). Avian communication in urban noise: causes and consequences of vocal adjustment. Auk, 123, 639-650. DOI: 10.1642/0004-8038(2006)123[639:ACIUNC]2.0.CO;2

Peris, S. J., \& Pescador, M. (2004). Effects of traffic noise on passerine populations in Mediterranean wooded pastures. Applied Acoustics, 65, 357-366. DOI: 10.1016/j.apacoust.2003.10.005

Potvin, D. A., Mulder, R. A., \& Parris, K. M. (2014). Silvereyes decrease acoustic frequency but increase efficacy of alarm calls in urban noise. Animal Behaviour, 98(1), 27-33. DOI: 10.1016/j.anbehav.2014.09.026

Raisbeck, G. (1963). Information Theory. Cambridge, Massachussets: MIT.

Ralph, C., Geupel, J., Geoffrey, R., Pyle, P., Martin, T. E., De Sante, D. F., \& Milá, B. (1996). Manual de métodos de campo para monitoreo de aves terrestres. Gen. Tech. Rep. PSW-GTR-159. Albany, CA: Pacific Southwest Research Station, Forest Service, U.S. Department of Agriculture.

Redondo, P., Barrantes, G., \& Sandoval, L. (2013). Urban noise influences vocalization structure in the House Wren Troglodytes aedon. Ibis, 155, 621-625. DOI: 10.1111/ibi.12053

Reijnen, R., Foppen, R., \& Veenbaas, G. (1997). Disturbance by traffic of breeding birds: evaluation of the effect and considerations in planning and managing road corridors. Biodiversity and Conservation, 6, 567-581. DOI: 10.1023/A:1018385312751 
Rheindt, F. E. (2003). The impact of roads on birds: does song frequency play a role in determining susceptibility to noise pollution? Journal of Ornithology, 144, 295-306. DOI: 10.1007/BF02465629

Ríos-Chelén, A., Salaberria, C., Barbosa, C., Macías-García, C., \& Gil, D. (2012). The learning advantage: bird species that learn their song show a tighter adjustment of song to noisy environments thestado an those that do not learn. Journal of Evolutionary Biology, 25, 2171-2180. DOI: 10.1111/j.1420-9101.2012.02597.x

Silva, M. L., Piqueira, J. C., \& Vielliard, J. M. E. (2000). Using Shannon entropy on measuring the individual variability in the rufous-bellied thrush Turdus rufiventris vocal communication. Journal of Theoretical Biology, 207, 57-64. DOI: 10.1006/jtbi.2000.2155

Slabbekoorn, H. (2004). Singing in the wild: the ecology of bird-song. En P. Marler \& H. Slabbekoorn (Eds.), Nature's music: the science of birdsong (pp. 178205). San Diego: Academic/Elsevier.

Slabbekoorn, H. (2013). Songs of the city: noise-dependent spectral plasticity in the acoustic phenotype of urban birds. Animal Behaviour, 85, 1089-1099. DOI: 10.1016/j.anbehav.2013.01.021

Slabbekoorn, H., \& Boer-Visser, A. (2006). Cities change the songs of birds. Current Biology, 16, 2326-2331. DOI: $10.1016 /$ j.cub.2006.10.008

Slabbekoorn, H., \& Peet, M. (2003). Birds sing at a higher pitching urban noise. Nature, 424, 267. DOI: $10.1038 / 424267 \mathrm{a}$
Slabbekoorn, H., \& Ripmeester, E. A. P. (2008). Birdsong and anthropogenic noise: implications and applications for conservation. Molecular Ecology, 17, 72-83. DOI: 10.1111/j.1365-294X.2007.03487.x

Specht, R. (1998). Avisoft SASLab Pro, Sound analysis and synthesis laboratory software for MS-Windows. Berlin, Alemania.

Suthers, R. A., Goller, F., \& Pytte, C. (1999). The neuromuscular control of birdsong. Philosophical Transactions of the Royal Society B: Biological Sciences, 354, 927-939. DOI: 10.1098/rstb.1999.0444

Weiserbs, A., \& Jacob, J. J. (2001). Is breeding bird distribution affected by motorway traffic noise? Alauda, 69, 483-489.

Wood, W. E. \& Yezerinac, S. M. (2006). Song sparrow (Melospiza melodia) song varies with urban noise. Auk, 123, 650-659. DOI: 10.1642/0004-8038(2006)123[650:SSM MSV]2.0.CO;2

Zande, V., Keurs, J., \& Weijden, W. J. (1980). The impact of roads on the densities of four bird species in an open field habitat-evidence for a long distance effect. Biological Conservation, 18, 299-321. DOI: 10.1016/0006-3207(80)90006-3

Zollinger, S., Podos, J., Nemeth, E., Goller, F., \& Brumm, H. (2012). On the relationship between, and measurement of amplitude and frequency in bird song. Animal Behaviour, 84, 1-9. DOI: 10.1016/j. anbehav.2012.04.026 